\title{
Recognition of Dynamic Video Contents With Global Probabilistic Models of Visual Motion
}

\author{
Gwenaëlle Piriou, Patrick Bouthemy, and Jian-Feng Yao
}

\begin{abstract}
The exploitation of video data requires methods able to extract high-level information from the images. Video summarization, video retrieval, or video surveillance are examples of applications. In this paper, we tackle the challenging problem of recognizing dynamic video contents from low-level motion features. We adopt a statistical approach involving modeling, (supervised) learning, and classification issues. Because of the diversity of video content (even for a given class of events), we have to design appropriate models of visual motion and learn them from videos. We have defined original parsimonious global probabilistic motion models, both for the dominant image motion (assumed to be due to the camera motion) and the residual image motion (related to scene motion). Motion measurements include affine motion models to capture the camera motion and low-level local motion features to account for scene motion. Motion learning and recognition are solved using maximum likelihood criteria. To validate the interest of the proposed motion modeling and recognition framework, we report dynamic content recognition results on sports videos.
\end{abstract}

Index Terms-Motion learning, motion recognition, probabilistic models, video analysis.

\section{INTRODUCTION}

$\mathbf{E}$ XPLOITING the tremendous amount of multimedia data, and specifically video data, requires to develop methods able to extract high level information related to semantic aspects. Video summarization, video retrieval, and video surveillance are examples of applications. These objectives may involve different video processing issues. The first one is video segmentation into relevant temporal units formed by grouping elementary shots. Detecting events of interest in video sequences represent another challenge, which usually relies on similarity techniques. Closely related to event detection, classifying dynamic content in videos is a third issue of key importance, which is often focused on human actions or gestures recognition. Inferring "concepts" from low-level video features is a highly challenging problem. The characteristics of a "semantic" event have to be expressed in terms of video primitives (color, texture, motion, shape, etc.) in a sufficiently discriminating way with respect to the contents to be processed [1].

In this paper, we address the problem of recognizing dynamic contents in videos. It can be formulated as inferring motion

Manuscript received April 8, 2005; revised April 6, 2006. This work was supported in part by the "Région Bretagne," in part by the IST European project LAVA, and in part by the European Network of Excellence MUSCLE. The associate editor coordinating the review of this manuscript and approving it for publication was Dr. Giovanni Poggi.

G. Piriou and P. Bouthemy are with the IRISA/INRIA, 35042, Rennes cedex, France (e-mail: gwenaelle.piriou@irisa.fr; patrick.bouthemy@irisa.fr).

J.-F. Yao is with the IRMAR, 35042 Rennes Cedex, France (e-mail: jian-feng. yao@univ-rennes1.fr).

Digital Object Identifier 10.1109/TIP.2006.881963 models or motion concepts from spatiotemporal low-level video features. We adopt a statistical approach involving motion modeling, (supervised) learning and classification issues. If specific motion classes were considered, such as rigid motion, human motion or dynamic textures, dedicated motion models could be used. They can be either explicitly derived from kinematic laws (e.g., 3-D kinematic screw for rigid motion [2], articulated motion for human motion [3]), or conveniently represented by simple parametric models (e.g., AR models for dynamic textures [4], 2-D polynomial models for camera effects [5]). Since we aim at dealing with general motion contents involving a large range of motion types, possibly in a combined way, predefined (specific or parametric) motion models are not sufficient anymore.

As a matter of fact, motion models have to be specified and learned from the image sequences themselves. Furthermore, we separate scene motion and camera motion and we introduce new global probabilistic motion models to represent them. These probabilistic models are exploited for learning dynamic content "concepts" and for detecting them in videos. The proposed framework is general. To validate it, we have considered sports videos involving dynamic contents which are complex enough while being unambiguously specifiable. Thus, the reported experiments are challenging while meaningful. Moreover, sports events can be easily mapped to image motion information in that context. Let us point out that the work described in this paper does not specifically deal with event detection in videos which often requires to combine different cues and modalities (audio and visual ones). We would rather focus on the modeling and recognition of motion contents. Motion is one of the main constituents (but not the only one) of a video event which can be defined as corresponding to a specific action (or as a sequence of elementary actions). In the sequel, we may nevertheless talk about event detection for convenience sake.

The remainder of this paper is organized as follows. Section II presents related work. Section III is concerned with the motionrelated measurements we use for motion modeling. Section IV is dedicated to the probabilistic modeling of motion information. The motion recognition algorithm is described in Section V. The learning process of dynamic content is detailed in Section VI. Experimental validation of the proposed probabilistic mixed-state motion models is discussed in Section VII, and results of the overall dynamic content detection framework on sports videos are reported in Section VIII. Finally, concluding remarks are given in Section IX.

\section{RELATED WORK}

Different approaches have been investigated for the characterization and the recognition of spatiotemporal video contents. 
Some of them are directly based on image intensities. In [6], Gaussian mixture models (GMMs) are used to represent color features and space-time locations and to provide a space-time segmentation of the video which is considered as the detection of dynamic regions. In [7], wavelet coefficients are directly estimated from the image sequence to characterize motion and the derived models are used for indexing tasks. In [8], temporal slices extracted from the volume of the image sequence, and directly obtained from MPEG data, are used to determine motion descriptors. These motion features combined with color features are exploited to group video shots using the K-means classification technique.

Motion information can be implicitly handled by space-time intensity gradients. In [9], histograms of spatial and temporal intensity gradients are exploited to cluster temporal dynamic events but involving a single actor and a static background only. In [10], local statistics obtained from spatiotemporal intensity gradients are computed around space-time interest points extracted in the image sequence, these points being characterized by significant local variations in both space and time. The designed recognition method relies on the matching of the spatiotemporal image descriptors using appropriate similarity measures.

More explicit motion features are also used for the characterization of spatiotemporal content. For example, optical flow is considered for human action change detection in [11]. However, the estimation of optical flow remains a difficult task in noncontrolled contexts. In [12] and [13], global motion characteristics are extracted from MPEG motion vectors for indexation purposes. However, the use of MPEG motion vectors is questionable, since it is really dependent on the MPEG encoder. Trajectory-based analysis is another domain of investigation for video characterization. Such methods are used in object-based indexing, retrieval tasks or for video surveillance applications. In [14], the authors present a classification method of motion trajectories using Gaussian mixture models and hidden Markov models. Tracking is, however, not that easy in most broadcast videos as sports TV programs.

A limitation of most of the methods involving numerical motion features is that a more or less empirical similarity measure is adopted involving the critical setting of thresholds. Moreover, they cannot properly cope with noisy measurements and variability (or uncertainty) in motion content.

Sports video analysis is also a popular objective due to the economical importance of sports TV programs and to future services to be designed in that context. Different approaches have been recently investigated to detect highlights in sports videos. Dominant color information is used in [15]. In [16], tools are proposed for content analysis of snooker videos by using motion detection and ball trajectories. In this context of highlight detection, most approaches are multimodal. In [17], the spectator excitement is modelled and derived from three variables related to audio-video features in order to extract the most interesting video segments (e.g., goals in soccer game). Methods developed in [18] and [19] are also concerned with selecting highlights in soccer games and used camera motion information combined with audio information, i.e., loundness [18], excited speech detected from pitch raise and energy increase [19]. In [20], the purpose is to detect significant events in broadcast
TV programs of american football games. To this end, the authors use textual and audio informations. More precisely, they exploit external metadata, called gamestats which are available in websites, and try to link video segments of the video stream with the description of the gamestats. In that vein of multimodal video analysis, in [21], the authors consider educational medical videos and propose a hierarchical classification method to classify video shots according to high-level semantic classes. Video shots are described by visual, auditory and textual descriptors and the classification steps are performed with Gaussian mixture models which represent the descriptors.

Hidden Markov models (HMMs) are widely used in the context of sports video analysis. Indeed, such a modeling is really suited in case of sports with specific structure associated with well-defined camera shooting rules. In [22], HMMs encoding players distribution and camera motion are used to discriminate three highlights: free kicks, corner kicks and penalty kicks. A similar approach of highlights recognition involving HMM is proposed in [23] for baseball videos. The considered observations also include descriptors related to the camera motion. The authors of [24] propose to identify the type of sport (soccer, hockey, football, or basketball) by learning two HMMs for each of them. In fact, color features are associated to the first HMM, while the second one is learned from the MPEG motion vectors. HMMs allow the characterization of other kinds of video too. In [25], the type of video scenes composed of several shots is identified among dialogues, stories, action and generics. Observations attached to the HMMs are both audio and motion features extracted from the MPEG stream. In [26], the goal is to classify videos in different program categories (commercial, weather forecast, football game, basketball game). Although HMMs are efficient for classification of videos, they are mainly applied to videos exhibiting specific structure. In some cases, the choice of the number of hidden states is not that straightforward. Moreover, the learning stage may be a long process since it requires manual indexing of numerous videos. In [27], instead of HMM, statistical models for components of the video structure such as shot duration and activity, are introduced to segment and to classify video sequences into different genres (sports, news, movies, etc.). More precisely, shot duration is modeled by an Erlang distribution or a Weibull one, while descriptors of activity can be represented by mixture models. Bayesian networks which convey a richer formalism than HMMs are also exploited for video analysis to identify the type of videos [28] or to classify motion [29], for example. Recently, in [30], dynamic Bayesian networks are compared for a classification task of soccer video segments into play and break events. One interest of the dynamic bayesian network is to provide a multilayer structure to account for complex events, even if layered HMM have also been proposed in [31].

In this paper, we focus on visual motion information and dynamic video contents. In particular, this is relevant to the detection of highlights or specific events in sports videos, since the latter can be easily mapped to motion information. We aim at processing a large range of dynamic contents as those encountered in TV sport programs. Here, we propose new probabilistic motion models. Such a modeling allows us to derive a parsimonious motion representation involving a small number of parameters, while coping with errors in the motion measurements 
and with significant variability in motion classes to be considered. We handle in a separate way the camera motion and the scene motion. The former can usually be related to the dominant image motion. As a consequence, scene motion can be related to residual image motion. Indeed, these two sources of motion bring important and complementary information, which have to be explicitly taken into account for dynamic content modeling and detection. As for motion measurements, we consider, on one hand, parametric motion models to capture the camera motion, and on the other hand, low-level local motion features to account for the scene motion. They convey more information than those used in [9], while still easily computable in contrast to optic flow. They can be efficiently and reliably computed in any video whatever its genre and its content.

To validate the interest of the proposed motion modeling framework, we have considered the recognition of dynamic contents in sports video. We have designed a two-step event detection method to restrict the recognition issue to a limited and pertinent set of classes, since probabilistic motion models have to be learned for each class of dynamic content to be recognized. A preliminary version of this work was described in [32]. The two-step framework allows us to simplify the learning stage, to save computation time and to make the overall detection more robust and efficient. The first step consists in selecting candidate segments of potential interest in the processed video. Typically, for sports videos, it comes to select the "play" segments. The second step is stated as a classification problem and amounts to recognize the relevant events (in terms of dynamic content) among the segments selected during the first step. It is solved using a maximum likelihood (ML) criterion.

\section{Motion MEASUREMENTS}

Let us first briefly describe the motion measurements that we use. Let us point out that the choice of these measurements is motivated by the goal we are pursuing, that is the recognition of important events in videos. This task is intended as a rather qualitative characterization which does not require a full estimation of the image motion.

It is possible to characterize the image motion as proposed in [33], by computing at each pixel a local weighted mean of the normal flow magnitude. However, as mentioned above, the image motion is actually the sum of two motion components: the dominant motion (which can be usually assumed to be due to camera motion) and the residual motion (which is then related to the independent moving objects in the scene, which will be referred to as the scene motion in the sequel). Thus, we first estimate the dominant image motion between successive images of the sequence. Then, we cancel the camera motion (i.e., we compensate for the estimated dominant image motion), which allows us to compute local motion-related measurements revealing the residual image motion only.

The dominant image motion is represented by a widely used deterministic 2-D affine motion model

$$
\mathbf{w}_{\theta}(p)=\left(\begin{array}{l}
a_{1}+a_{2} x+a_{3} y \\
a_{4}+a_{5} x+a_{6} y
\end{array}\right)
$$
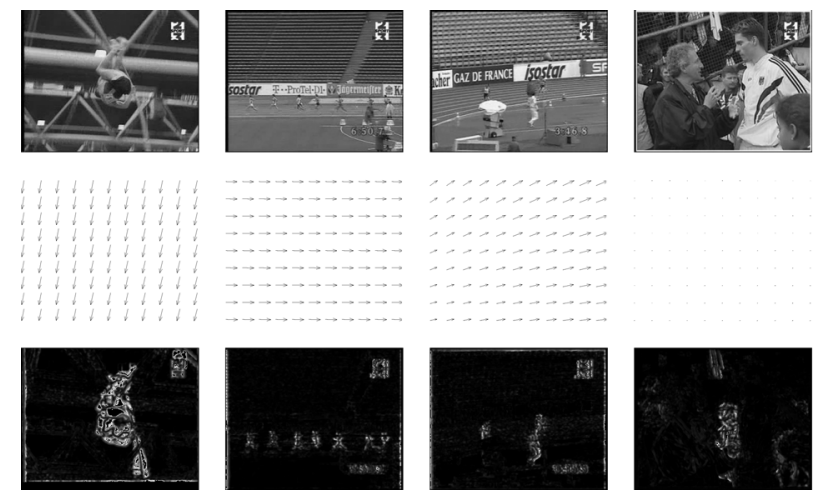

Fig. 1. Athletics video. Top row: Four images at different time instants of the Athletics video involving, respectively, an upward-tilt camera motion, a left panning one, a zoom combined with a panning motion, and a static shot. Middle row: Corresponding maps of the estimated dominant motion fields. Bottom row: residual motion measurements $v_{\text {res }}$ (zero-value in black).

where $\theta=\left(a_{i}, i=1, \ldots, 6\right)$ is the model parameter vector and $p=(x, y)$ is an image point. This simple motion model can correctly handle different camera motions such as panning, zooming, tracking, (including, of course, static shots). To compute this camera motion model, we use the robust real-time multiresolution estimation method ${ }^{1}$ we have described in [34]. Let us point out that the motion model parameters are directly computed from the spatiotemporal derivatives of the intensity function. Thus, the camera-motion flow vector $\mathbf{w}_{\hat{\theta}_{t}}$ can be derived at each time $t$ and for each pixel $p$ once the estimated motion parameters $\hat{\theta}_{t}$ are available, using (1).

Furthermore, the residual motion measurement $v_{\text {res }}(p, t)$ is defined as the weighted local mean of the normal residual flow magnitudes $\left|v_{n}\right|$, the weights being given by the square of the magnitude of the spatial intensity gradient. This weighted averaging allows us to smooth out the noise attached to the computation of the normal flow and to enforce the reliability of the motion measurements. We finally get

$$
v_{\mathrm{res}}(p, t)=\frac{\sum_{q \in \mathcal{F}(p)}\|\nabla I(q, t)\|^{2} \cdot\left|v_{n}(q, t)\right|}{\max \left(\eta^{2}, \sum_{q \in \mathcal{F}(p)}\|\nabla I(q, t)\|^{2}\right)}
$$

with $v_{n}(q, t)=\left(I(q, t)-I\left(q+w_{\hat{\theta}_{t}}(q), t+1\right)\right) /\|\nabla I(q, t)\|$. Here, $\mathcal{F}(p)$ is a local spatial window centered in pixel $p$ (typically, a $3 \times 3$ window), $\nabla I(q, t)$ the spatial intensity gradient of pixel $q$ at time $t$, and $\eta^{2}$ a predetermined constant related to the noise level. Such measurements have already been successfully used for the detection of independent moving objects in case of a mobile camera [35], [36]. In practice, an upper bound $v_{\max }$ is introduced for the local residual motion measurements. More precisely, local residual motion measurements over $v_{\max }$ are ignored since large displacements cannot be handled with these measurements. A way to handle large displacements is to follow a multiresolution approach to compute the normal flow as proposed in [33]. In this work, since we are only considering global occurrences of the (weighted) normal flow magnitudes,

${ }^{1}$ The corresponding software (called Motion-2D) is available and can be downloaded at http://www.irisa.fr/vista/Motion2D. 
it turned out that it was not necessary to include this supplementary (time-consuming) computation step.

Fig. 1 displays images of an athletics TV program with respectively the maps of the estimated dominant motion vectors $\mathbf{w}_{\theta}$ and the maps of residual motion measurements $v_{\text {res }}$. These examples show that the camera motion is reliably captured even in case of multiple moving elements in the scene. It also indicates that the scene motion is correctly accounted for by the residual motion measurements. Let us mention that residual motion is present on logo areas when the camera is moving, and it is null when the camera is static (fourth example) as expected.

From relation (2), it can be straightforwardly noted that we only get information related to local motion magnitude, and, consequently, we discard the local motion direction. As demonstrated by the results reported later, this is not a shortcoming since we aim at detecting events, i.e., at determining "qualitative" motion classes. On the other hand, the orientation of the normal flow vector is nothing but the orientation of the spatial image gradient which is of course independent of motion. Besides, a motion direction information is nevertheless exploited through the camera motion velocity vectors. As for sports videos, they indirectly convey information on the scene motion detection as well, since the camera usually follows the players or the athlets along the shots.

\section{Probabilistic Modeling of Motion}

The proposed method for detecting dynamic events relies on the probabilistic modeling of the motion content in a video. Indeed, the large diversity of video contents leads us to favor a probabilistic approach which moreover allows us to formulate the problem of event recognition within a Bayesian framework. Due to the different, but complementary, nature of the information brought by the residual motion (or scene motion) and by the dominant motion (or camera motion), two different probabilistic models have to be defined for these two motion components. We search for models involving as few parameters as possible while retaining the main characteristics of the motion content for classification purpose. In contrast, the model we have introduced in [33] still involved up to one hundred parameters and required to deal with quantized motion measurements to evaluate co-occurrence statistics.

\section{A. Residual Motion}

We first describe the probabilistic model of scene motion derived from statistics on the local residual motion measurements expressed by relation (2). The 1-D histograms of these measurements computed over different video segments reveal two degrees of freedom as illustrated on the histograms of Fig. 7. They usually exhibit a prominent peak at zero and a continuous component part which can be modeled by a distribution from an exponential family. More formally, we can state that the variable $v_{\text {res }}$ is a mixed-state variable, since it can take both discrete values, here the singleton $\{0\}$ which can be assimilated to a symbolic value (here, no motion), and continuous values over $(0, \infty)$ accounting for the actual motion measurements. There- fore, we model the distribution of the local residual motion measurements within a video segment by a specific mixture model which is called mixed-state model. with density

$$
f(z)=\alpha \delta(z)+(1-\alpha) \delta^{*}(z) \phi_{\beta}(z)
$$

where $z$ holds for $v_{\text {res }}(p, t), \alpha$ is the mixture weight, $\delta$ denotes the indicator function at $0: \delta(z)=\mathbf{1}_{\{0\}}(z)$ and $\delta^{*}(z)=$ $1-\delta(z)$. Here, $\phi_{\beta}$ designates the density of the continuous distribution. The density $f$ is defined w.r.t the measure $\mu$

$$
\mu(d z)=\delta_{0}(d z)+\lambda(d z)
$$

where $\delta_{0}$ denotes the Dirac measure at 0 and $\lambda$ represents the Lebesgue measure.

We have explored two types of distribution for $\phi_{\beta}$. The first one is the Gaussian distribution (restricted to positive values) with density $\phi_{\beta}(z)=(2 / \sqrt{2 \pi} \beta) \exp -\left(z^{2} / 2 \beta^{2}\right)$ for $z>0$. The mixture model defined by the density $f$ is then called Gaussian mixed-state model. The second one is the exponential distribution with density $\phi_{\beta}(z)=(1 / \beta) \exp -(z / \beta)$ for $z>0$. In this case, we name it exponential mixed-state model.

The two parameters $\alpha$ and $\beta$ are estimated using the ML criterion. Let $\{z(p, t)\}_{(p, t) \in S \times[1, T]}$ denote the set of observations, where $S$ represents the image grid and $T$ is the number of frames of the video segment. Let

$$
\begin{gathered}
n_{0}=\#\{(p, t) \in S \times[1, T] ; z(p, t)=0\} \\
n_{+}=\#\{(p, t) \in S \times[1, T] ; z(p, t) \neq 0\} .
\end{gathered}
$$

Then, $\alpha$ is estimated by

$$
\hat{\alpha}=\frac{n_{0}}{n_{0}+n_{+}} .
$$

If $\phi_{\beta}$ is the density of the restricted Gaussian distribution, $\beta$ is estimated by

$$
\hat{\beta}^{2}=\frac{1}{n_{+}} \sum_{(p, t) \in S \times[1, T]} z^{2}(p, t) .
$$

If $\phi_{\beta}$ is the density of the exponential distribution, $\beta$ is estimated by

$$
\hat{\beta}=\frac{1}{n_{+}} \sum_{(p, t) \in S \times[1, T]} z(p, t) .
$$

Let us recall that, in practice, local residual motion measurements $z(p, t)$ which are over $v_{\max }$ are ignored. Here, $v_{\max }=8$.

In order to capture not only the instantaneous motion information, but also its temporal evolution over the video segment, the temporal contrasts $\Delta v_{\text {res }}$ of the local residual motion mea- 
surements are also considered. They are defined as the temporal difference of the variables $v_{\text {res }}$

$$
\Delta v_{\text {res }}(p, t)=v_{\text {res }}(p, t+1)-v_{\text {res }}(p, t) .
$$

They are modeled by a mixture $g\left(z^{\prime}\right)$ of the indicator function at 0 and a zero-mean Gaussian distribution, where $z^{\prime}$ holds for $\Delta v_{\text {res }}(p, t)$. The mixture weight and the variance of the Gaussian distribution are still evaluated using the ML criterion and their expressions are similar to (7) and (8).

The density of the full probabilistic residual motion model is then simply defined as the product of these two densities as follows:

$$
h^{\mathrm{res}}\left(z, z^{\prime}\right)=f(z) \cdot g\left(z^{\prime}\right) .
$$

Considering the product of the two densities is a simple choice to combine the two variables $v_{\text {res }}$ and $\Delta v_{\text {res }}$ in the same model. A more sophisticated model could be considered instead, to account for possible correlation. Nevertheless, we prefer considering a simple product model which allows us to add and combine information with lower cost.

The probabilistic residual motion model is completely specified by four parameters which can be easily estimated. It accounts for global statistics accumulated over space (image grid) and time (i.e., over all the frames of the video segment). It can be considered as a global occurrence model. Obviously, this model does not allow us to capture how the motion information is spatially distributed in the image plane, but this is not necessary for the objective we consider here.

\section{B. Dominant Image Motion}

We have to design a probabilistic model of the camera motion to combine it with the probabilistic model of the residual motion in the recognition process. We first investigated the characterization of the camera motion using the affine motion model parameter vector $\theta$ defined in Section III and we represented its distribution over the video segment by a probabilistic model. The main difficulty in that case is to propose a valid probabilistic model. Indeed, if the distribution of the two zero-order parameters $a_{1}$ and $a_{4}$ can be easily inferred (these two parameters are likely to be constant within a video segment so that a Gaussian mixture could reasonably be used to account for the distribution of these parameters over a given event class), the task becomes more difficult when dealing with the other parameters which may be not constant anymore over a segment and are not of the same nature.

We have proposed instead to consider the equivalent representation supplied by the 2-D motion vectors $w_{\theta_{t}}(p, t)$, and to exploit them as a 2-D histogram [37]. More precisely, at each time $t$, the motion parameters $\theta_{t}$ of the camera motion model (1) are estimated and the vectors $w_{\hat{\theta}_{t}}(p)$ are computed for each point $p$ of the image support (a subsampled image grid could be used too) at each time instant $t$ of the video segment. The values of the horizontal and vertical components of $w_{\hat{\theta}_{t}}(p, t)$ are then finely quantized, and we form the empirical 2-D histogram of their distribution over the considered video segment. Finally, this histogram is modeled by a standard mixture $\gamma^{\text {cam }}$ of 2-D Gaussian distributions. The number of components of the mixture is determined with the Integrated completed Likelihood Criterion (ICL) [38] and the mixture model parameters are estimated using the expectation-maximization algorithm.

\section{RECOGNITION OF DyNAMIC VIDEO CONTENTS}

We now exploit the designed probabilistic motion models for the problem of recognizing dynamic contents in video. Learning the "concepts" of dynamic content to be involved in an event detection task will turn out to estimating the parameters of these models for each specified class of dynamic video content.

We suppose that the videos to be processed are segmented into temporal units. This preliminary step is out of the scope of this paper which focuses on the motion modeling, learning and recognition issues. To segment the video, we can use either a shot change detection technique [5], a camera-motion segmentation method, or both in a combined way [19]. We choose this last option. Let $\left\{s_{i}\right\}_{i=1, \cdots, N}$ be the partition of the processed video into temporal segments. Due to the temporal video segmentation we used (including the detection of shot changes and camera motion changes), the temporal segments are supposed to be homogeneous with respect to both the dominant motion (by definition of the video segmentation algorithm) and the residual motion (due to the shooting rules for sports broadcasts).

\section{A. Selecting Video Segments}

The first step of our dynamic content recognition method consists in dividing the video segments in two groups. The first group includes the segments likely to contain the searched events, the second one is formed by the video segments to be definitively discarded. Typically, for sports videos, we try to first distinguish between "play" and "no play" segments. This step is based only on the residual motion which accounts for the scene motion. Therefore, only single-variable probabilistic models are used, which saves computation time. To this end, several motion models are learned offline in a training stage for each of the two groups of segments, since each group is supposed to contain video segments with different motion contents. This will be detailed in Section VI. Let us denote $\left\{h_{1, n}^{\text {res }}, 1 \leq n \leq N_{1}\right\}$ the densities of the residual motion models learned for the "play" group and $\left\{h_{2, n}^{\text {res }}, 1 \leq n \leq N_{2}\right\}$ the densities of those learned for the "no play" group. Then, the sorting consists in assigning the label $\zeta_{i}$, whose value can be 1 for "play" or 2 for "no play," to each segment $s_{i}$ of the processed video using the ML criterion defined as follows:

$$
\zeta_{i}=\underset{k=1,2}{\arg \max }\left[\max _{1 \leq n \leq N_{k}} \prod_{(p, t) \in s_{i}} h_{k, n}^{\mathrm{res}}\left(z(p, t), z^{\prime}(p, t)\right)\right]
$$

\section{B. Detecting the Relevant Dynamic Contents}

1) Problem Statement: The second step of the proposed method effectively deals with the detection of the dynamic 
contents of interest within the previously selected segments. Contrary to the first step, the two kinds of motion information (scene motion and camera motion) are simultaneously exploited, since their combination permits to more precisely characterize a specific event. An offline training stage is required to learn the dynamic content concepts involved in the event detection task. As explained in Section VI, a residual motion model with density $h_{j}^{\text {res }}$ and a camera motion model with density $\gamma_{j}^{\text {cam }}$ have to be estimated from a given training set of video samples, for each event $j$ to be retrieved. The detection is performed in two substeps. First, we assign to each preselected segment the label of one of the dynamic contents classes introduced in the considered task. This issue is stated as a classification problem which avoids the need of detection thresholds and allows all the considered events to be extracted in a single process. Since wrong segments might be included in the preselected segments, a validation step is then applied to confirm or not the assigned labels.

2) Video Segment Labeling: We consider only the segments $s_{i}$ which have been selected as "play" segments during the first step described above. The video segments are labeled with one of the $J$ learned classes of dynamic contents according to the ML criterion. More precisely, the label $\xi_{i}$ assigned to the segment $s_{i}$ takes its value in the label set $\{1, \ldots, J\}$. Let $L_{j}\left(s_{i}\right)$ be the conditional likelihood of the segment $s_{i}$ subject to the learned models for class $j$. It is defined by

$L_{j}\left(s_{i}\right)=\prod_{(p, t) \in s_{i}} h_{j}^{\mathrm{res}}\left(z(p, t), z^{\prime}(p, t)\right) . \prod_{(p, t) \in s_{i}} \gamma_{j}^{\mathrm{cam}}\left(w_{\hat{\theta}_{t}}(p)\right)$.

Thus, the label $\xi_{i}$ of the segment $s_{i}$ is given by

$$
\xi_{i}=\underset{j=1, \ldots, J}{\arg \max } L_{j}\left(s_{i}\right)
$$

Let us note that prior on the classes could be easily introduced in (14) leading to a MAP criterion.

3) Class Label Validation: By applying (14), we can label all the segments supplied by the first selection step. However, we have to consider that there might be "no play" segments wrongly labeled as "play" after the first selection step. We call them "outliers." These segments are forced to be assigned one of the event classes using relation (14), which creates false detection. As a consequence, we propose a validation test, involving only residual motion models. It consists in testing for each segment $s_{i}$ the hypotheses defined by

$$
\left\{\begin{array}{c}
H_{0}: \quad " \quad s_{i} \text { really belongs to the class } l_{i} \\
\quad \text { determined by }(14) " \\
H_{1}: \quad " \quad s_{i} \text { is labeled as } l_{i} \text {, whereas it is } \\
\text { an outlier segment." }
\end{array}\right.
$$

To this end, a set of models with densities $\bar{h}_{j}^{\text {res }}$ has to be specified and estimated to represent the outlier segments. This will be explained in Section VI.
The likelihood test between these two hypotheses can be formulated as follows:

$$
\begin{array}{ll}
\text { if } \frac{\bar{L}_{j}^{\text {res }}\left(s_{i}\right)}{L_{j}^{\text {res }}\left(s_{i}\right)}>\varepsilon, & H_{1} \text { is accepted } \\
\text { else, } & H_{0} \text { is accepted }
\end{array}
$$

where $L_{j}^{\text {res }}\left(s_{i}\right)$ is the likelihood of the video segment $s_{i}$ related to the residual motion model which is learned for motion event $j$. Similarly, $\bar{L}_{j}^{\text {res }}\left(s_{i}\right)$ is the likelihood of the video segment $s_{i}$ related to the outliers residual model defined by the density $\bar{h}_{j}^{\text {res }}$. In this way, we can correct some misclassifications resulting from the imperfect output of the first selection step, by discarding the video segments which are rejected by the likelihood test (15).

\section{LEARNING THE MOTION MODELS}

For a given video genre (e.g., TV programs of athletics, skating or tennis games), a training step is performed offline in order to learn the residual motion models and the dominant motion models needed by the dynamic content recognition method. Let us point out that we have to divide the training set in two subsets. The first one is used to learn the motion models required by steps 1) and 2) of the motion recognition algorithm, while the second one allows us to learn the outlier motion models. Let us recall that our models for motion information are highly motivated by the need to build relatively low-dimensional models while achieving an acceptable performance for the target recognition task. All these models have less than ten parameters. As a comparison, standard models for motion measurements based on co-occurrence statistics involve several hundreds parameters. On the other hand, we have a huge amount of data (usually several minutes of video of $288 \times 352$ sized views). Consequently, at this stage, we do not really incur the risk of overfitting.

\section{A. Learning the Residual Motion Models for the Initial Selection Stage}

As the first selection stage involves the scene motion only, we have to learn residual motion models as specified in (IV-A). Because of the large diversity of video contents in the two groups "play" and "no play," we have to represent each group with several scene motion models. We apply the ascendant hierarchical classification (AHC) technique, separately to the "play" group and to the "no play" group of the training set. The overall procedure is defined as follows (for a given group).

- Step 0: A residual motion model is estimated for each video segment belonging to the training set of the considered group. At this early stage, each segment forms a cluster.

- Step 1: The two clusters (either composed of one segment or of several segments) found as the nearest ones w.r.t the symmetrized Kullback-Leibler distance between their corresponding residual motion models, are merged in the same cluster. The expression of this distance between two residual motion models with densities $h_{j}^{\text {res }}$ and $h_{k}^{\text {res }}$ is

$d\left(h_{j}^{\mathrm{res}}, h_{k}^{\mathrm{res}}\right)=\frac{1}{2}\left(d_{\mathrm{KL}}\left(h_{j}^{\mathrm{res}}, h_{k}^{\mathrm{res}}\right)+d_{\mathrm{KL}}\left(h_{k}^{\mathrm{res}}, h_{j}^{\mathrm{res}}\right)\right)$ 
where

$$
d_{\mathrm{KL}}\left(h_{j}^{\mathrm{res}}, h_{k}^{\mathrm{res}}\right)=d_{\mathrm{KL}}\left(f_{j}, f_{k}\right)+d_{\mathrm{KL}}\left(g_{j}, g_{k}\right) .
$$

The expression of the Kullback-Leibler distance between the density functions $f_{j}$ with parameters $\left(\alpha_{j}, \beta_{j}\right)$, and $f_{k}$ with parameters $\left(\alpha_{k}, \beta_{k}\right)$, of the residual motion measurements is given by

$$
d_{\mathrm{KL}}\left(f_{j}, f_{k}\right)=\int \ln \left(\frac{f_{j}(z)}{f_{k}(z)}\right) f_{j}(z) \mu(d z) .
$$

Let us remember that the measure $\mu$ is defined by (4). If $f_{j}$ and $f_{k}$ are densities of a positive Gaussian mixedstate model [i.e., the function $\phi$ in (3) is the density of the Gaussian distribution restricted to positive values], we have

$$
\begin{aligned}
d_{\mathrm{KL}}\left(f_{j}, f_{k}\right)=\alpha_{j} \ln \left(\frac{\alpha_{j}}{\alpha_{k}}\right) & +\frac{1-\alpha_{j}}{2}\left(\frac{\beta_{j}^{2}}{\beta_{k}^{2}}-1\right) \\
& +\left(1-\alpha_{j}\right) \ln \left(\frac{\beta_{k}\left(1-\alpha_{j}\right)}{\beta_{j}\left(1-\alpha_{k}\right)}\right)
\end{aligned}
$$

and if $f_{j}$ and $f_{k}$ are densities of an exponential mixedstate model [i.e., the function $\phi$ in (3) is the density of the exponential distribution], we get

$$
\begin{array}{r}
d_{\mathrm{KL}}\left(f_{j}, f_{k}\right)=\alpha_{j} \ln \left(\frac{\alpha_{j}}{\alpha_{k}}\right)-\left(1-\alpha_{j}\right)\left(\frac{\beta_{j}+\beta_{k}}{\beta_{k}}\right) \\
+\left(1-\alpha_{j}\right) \ln \left(\frac{\beta_{k}\left(1-\alpha_{j}\right)}{\beta_{j}\left(1-\alpha_{k}\right)}\right) .
\end{array}
$$

The Kullback-Leibler distance between the density functions $g_{j}$ and $g_{k}$ of the temporal contrasts can be similarly written as (19).

A residual motion model is then estimated for the new resulting cluster. We iterate until the stopping criterion is satisfied.

- Stopping criterion: We stop if the maximum of the symmetrized Kullback-Leibler distances between two clusters is lower than a certain percentage of the maximum of the distances computed at step 0 .

At this stage, the load of manually labelling the video segments of the training set is kept low. Indeed, we just need to sort the video segments into the two groups: "play" and "no play." At the end, each group is represented by a (small) set of clusters (depending on the heterogeneity of the video segment set) and their associated residual motion models, both obtained in an automatic way.

\section{B. Learning the Motion Models of the Dynamic-Content Classes}

Camera motion models and residual motion models representing the different dynamic-content classes to be recognized are required for the second stage of our recognition method. We first need a manual labelling of the "play" segments of the training set according to the dynamic-contents to detect.
For each dynamic-content class, a camera motion model is estimated from the video segments representing the considered class as explained at the end of Section IV-B. Similarly, the four parameters of the residual motion models for each class are estimated using the ML criterion.

\section{Learning of Outlier Motion Models}

We have also to determine probabilistic motion models to represent the outlier segments. To this end, we exploit the second set of training videos. It is important to consider a different set of video segments than the one used to learn the models involved in the first two stages of the recognition method. The selection stage into "interesting" and "non interesting" (or "play" "no play") groups is first applied to this second subset of the training set. The outlier segments are then determined (since we have the ground truth on that training set) and submitted to the classification stage of the method. Finally, we get a subset of outlier segments associated to each predefined event $j$, which allows us to estimate the associated residual motion model previously denoted by $\overline{\mathcal{M}}_{\text {res }}^{j}$.

\section{EXPERIMENTAL VALIDATION OF the PROBABiListic MODEL OF RESIDUAL MOTION}

Let us stress that, for all experiments, a thresholding step is added to assess the zero nature of the local residual motion measurement $v_{\text {res }}$. Indeed, the computed $v_{\text {res }}$ are rarely strictly equal to zero due to the numerical computation involved and the presence of noise. The threshold is set to 0.1. The same holds for the temporal difference $\Delta v_{\text {res }}$ of $v_{\text {res }}$.

\section{A. Experimental Comparison With a Histogram-Based Technique}

Concerning the analysis of the residual motion, we have carried out an experimental comparison between our statistical approach and a histogram-based technique since we are exploiting occurrence statistics, namely histograms. We consider the same local motion measurements for the histogram technique. We utilize three histograms: the histogram of residual motion measurements $v_{\text {res }}$ (2), the histogram of their temporal contrasts $\Delta v_{\text {res }}$ (10), and the 2-D histogram of the camera-motion flow vectors (1). Each class $j$ is then represented by three histograms: $H_{v_{\text {res }}}^{j}$, $H_{\Delta v_{\text {res }}}^{j}$ and $H_{\text {cam. }}^{j}$. The histograms are computed with a quantization step of 0.1 .

To compare two histograms, we consider the Euclidian distance, denoted by $d_{1}$ for 1-D histograms and by $d_{2}$ for 2-D histograms. We are aware that several distances can be considered to compare two histograms, and that this issue has to be carefully addressed. However, the computed motion measurements are all real values and we have a huge number of available computed values. Accordingly, we can consider a very fine quantization and the resulting histograms are very close to the real continuous distributions. Moreover, the histogram distance is only used to rank the classes. The Euclidean distance is then a reasonable choice.

These histograms are computed (and stored) for each event $j$ from the training set of video samples. Then, we consider the test set and we compute the three histograms $H_{v_{\text {res }}}^{s_{i}}, H_{\Delta v_{\text {res }}}^{s_{i}}$ and 

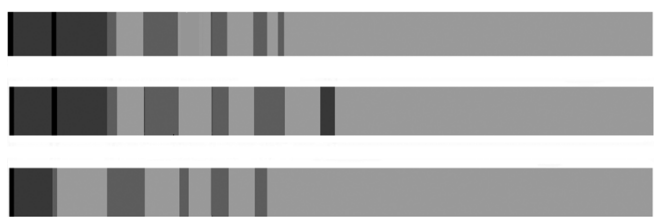

Fig. 2. Athletics video. Detection of four events. Top row: Ground truth. Middle row: Results obtained with the probabilistic motion models. Bottom row: Results obtained with the histogram-based technique. From dark to bright: Pole vault, replay of pole vault, long shot of track race, and closeup of track race.

$H_{\text {cam }}^{s_{i}}$, for each video segment $s_{i}$ of the test set. The classification step is now formulated as assigning the label $l_{i}$ of the event which minimizes the sum of the distances between histograms

$$
\begin{aligned}
l_{i}=\arg \min _{j=1, \ldots, J}\left(d_{1}\left(H_{v_{\text {res }}}^{s_{i}}, H_{v_{\text {res }}}^{j}\right)\right. & \\
& \left.\quad+d_{1}\left(H_{\Delta v_{\text {res }}}^{s_{i}}, H_{\Delta v_{\text {res }}}^{j}\right)+d_{2}\left(H_{\text {cam }}^{s_{i}}, H_{\text {cam }}^{j}\right)\right) .
\end{aligned}
$$

In order to focus on the classification performance of the two methods, the test set only involves "play" segments.

We have processed a part of an athletics TV program which includes jump events and track race shots. Some representative images of this video are displayed on Fig. 1. We want to recognize four events: Pole vault, Replay of pole vault, Long shots of track race and Closeup of track race. Consequently, we have to learn four residual motion models and four camera motion models for the method based on the probabilistic motion modeling. In Fig. 2, the processed video is represented by a time line exhibiting the duration of the video segments. Only segments of these four classes have been retained and concatenated to form the time line. A color is associated to each class. The first row corresponds to the ground truth, the second one and the third one contain the results obtained using the probabilistic framework and the histogram technique respectively. These results demonstrate that the probabilistic framework yields quite satisfactory results and outperforms the histogram-based technique.

\section{B. Unsupervised Clustering of a Video Database}

We report here results of the ascendant hierarchical classification (AHC) applied to a set of different videos for the residual motion only. The purpose is to assess the discrimination performance of our probabilistic residual motion modeling by considering an unsupervised classification task. We have processed a set of twenty short videos. There are two TV news videos, two other videos of low-level activity (Hall and Meeting), two highway scenes, two videos of takeoff and landing of a plane, and finally twelve sports videos including soccer, windsurf, hockey and basketball. The median images of the video segments are displayed on Fig. 3, except for the two TV news videos for copyright constraint.

Fig. 4 shows the binary tree obtained by the ascendant hierarchical classification technique. The values of the symmetrized Kullback-Leibler distances between the merged clusters are given at each node of the tree. By considering the six clusters obtained with a sufficiently low distance (i.e., here with distance $<0.15$ ), interesting observations can be deduced. The first cluster is formed by news ${ }_{1}$, news 2 , and hall which are all videos with very low-level activity. The second cluster is composed of three videos of intermediate-level activity (meeting, plane 1 , and plane 2 ). The three soccer videos belong to the same cluster. At this stage of the algorithm, the video basket $_{1}$ forms a single cluster. Contrary to the two other basketball videos which are closeup of players, this one involves a wide view of the play ground. This explains that it is further merged with the long shots of soccer games in the sequel of the ascendant classification. The fifth cluster contains closeup of

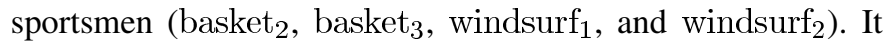
happens rarely that two videos of similar motion contents are not in the same cluster and, conversely, that a given cluster is not so homogeneous. The sixth cluster is formed by the two videos of highway scenes and the hockey video segments.

Let us stress that, here, the semantic interpretation have to be formulated only from visual motion (and not from objects, environments, or type of sports) since we are only dealing with motion information. Besides, this motion modeling is intended to be used in a supervised way in practice. Finally, this experiment has demonstrated that the proposed motion modeling has a quite satisfactory capacity of discriminating different motion contents while being compact and easy to compute.

\section{EXPERIMENTAL RESULTS OF THE DYNAMIC-CONTENT RECOGNITION METHOD}

We report experimental results obtained on sports videos. The aim is to validate our motion modeling and recognition framework by detecting dynamic contents of interest in these videos. Examples of events related to these dynamic contents are instances of pole vault or track race in an athletics meeting, or rally instances in a tennis game.

\section{A. Skating Videos}

The goal in this experiment dealing with skating videos is to distinguish between "play" segments which correspond to skating (simple skating motion, artistic effects, dance movements) and "no play" segments involving shots such as views of the audience, skaters waving at the beginning and at the end of each show, skaters waiting for the scores, as illustrated in Fig. 5. Two performances $(23 \mathrm{~min}$ ) of the first skating video are used as the training set. In the first experiment, the last performance (9 min) of the same TV program is used as the test set.

The precision $P$ and the recall $R$ for the "play" group are defined as follows:

$$
\begin{aligned}
P & =\frac{\# \text { correct }}{\# \text { correct }+\# \text { outliers }} \\
R & =\frac{\# \text { correct }}{\# \text { correct }+\# \text { missed }}
\end{aligned}
$$

where \#correct is the number of video segments labeled as "play" segments which effectively belong to this group, \#outliers is the number of "no play" segments wrongly labeled as "play" segments, and \#missed is the number of "play" segments labeled as "no play" segments. For this first experiment, the obtained precision was 0.85 and the recall 0.89 . Let us point out that this video is not so easy to process (large 


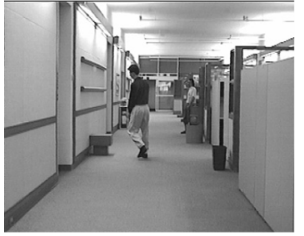

Hall

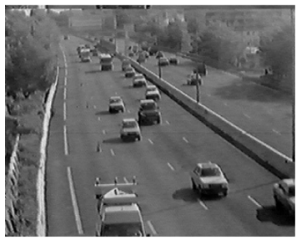

Highway $_{1}$

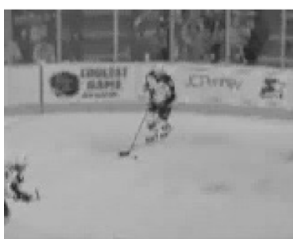

Hockey $_{1}$

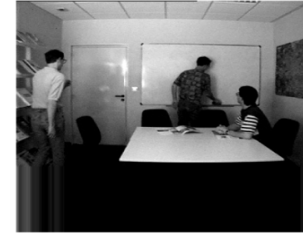

Meeting

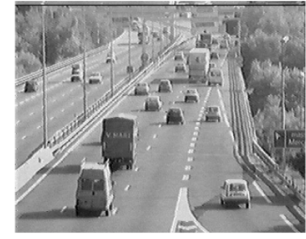

Highway $_{2}$

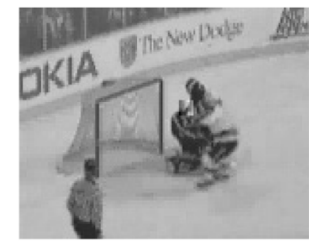

Hockey $_{2}$

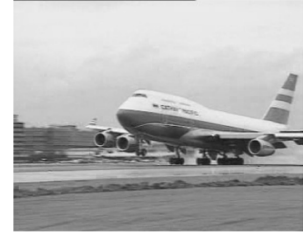

Plane $_{1}$

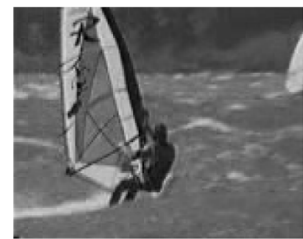

Windsurf $_{1}$

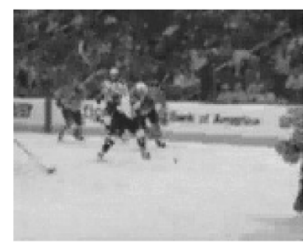

Hockey $_{3}$

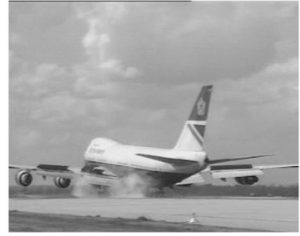

Plane $_{2}$

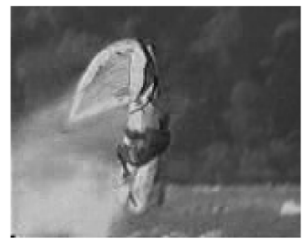

Windsurf $_{2}$

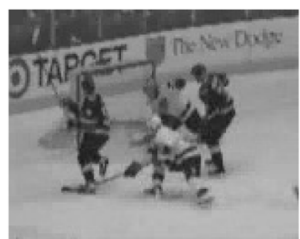

Hockey $_{4}$

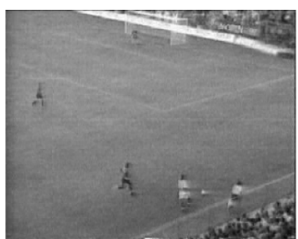

Soccer $_{1}$

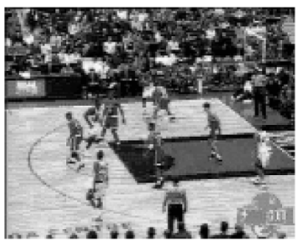

Basket $_{1}$

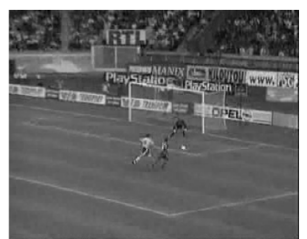

Soccer $_{2}$

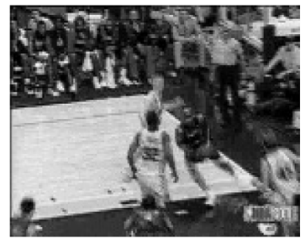

Basket $_{2}$

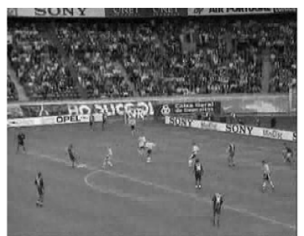

Soccer $_{3}$

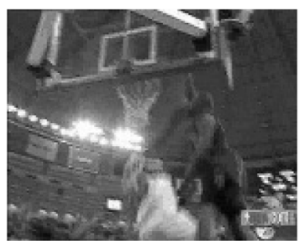

Basket $_{3}$

Fig. 3. Median images of the video segments which form the database.

displacements are frequent, the ice surface forms large uniform areas with specular effects, etc.).

We applied the play/no play selection stage to four other skating TV programs while keeping the same residual motion models learned from the first video. Each row of Fig. 6 contains images extracted from one of the four test videos. It can be observed these four videos are quite different from the training one. Indeed, the training video involves only "dance" performances, while the other four videos contain "ladies," "men," "pairs," and "dance" performances. Moreover, these four videos correspond to other championships than the one of the training set. The numbers of images of each processed video sequences are mentioned in Table I. As shown in Table I, satisfactory results are obtained for each processed video, especially for the precision rates. Recall rates are less high, due to the fact that the training video cannot account for all the variability of the test video contents.

\section{B. Athletics Video}

We have then applied our algorithm to the athletics TV program introduced in Section VII-A. We consider the following problem: detecting pole vault events, long shots of track race and closeups of track race in the video. The first $10 \mathrm{~min}(15000$ images) form the training set and the test set consists of the last 7 min (10 500 images). Since it is a rather short video, no outlier models are learned.

The first step of our method consists in separating the "play" segments which involve here pole vault and track race shots and the "no play" segments containing interview shots, large views of the stadium and replay of pole vault.

For each class to detect, one residual motion model and one camera motion model are learned from the training set for the second step of the algorithm. Histograms of the residual motion measurements and of the temporal contrasts of the residual 


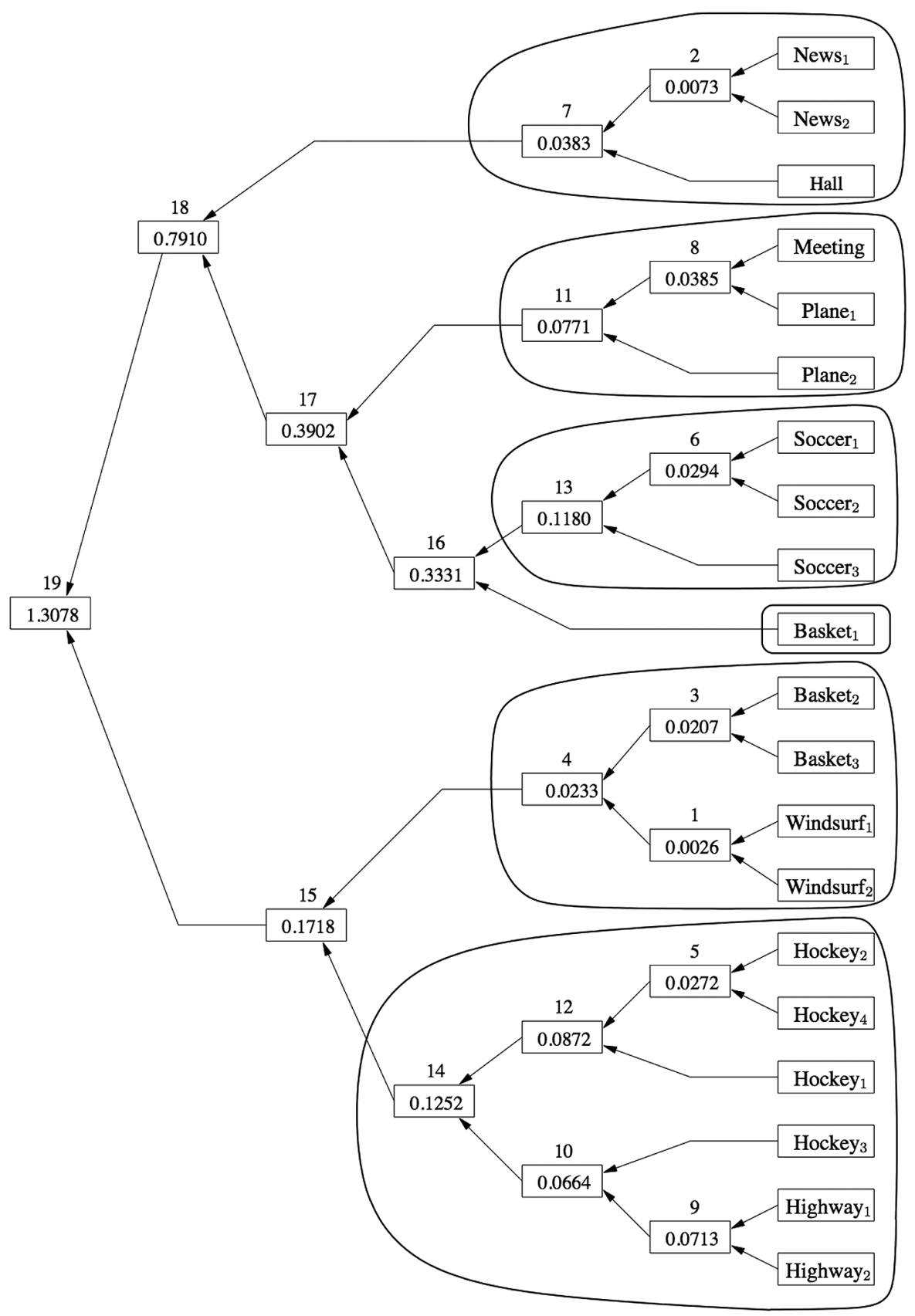

Fig. 4. Binary tree obtained by the ascendant hierarchical classification on the video set of Fig. 3. The blobs are the six clusters obtained with low distance.
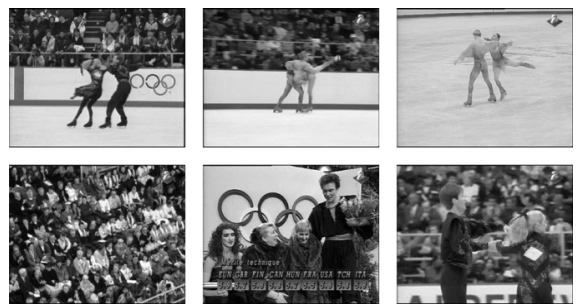

Fig. 5. Skating video. Images extracted from the video used as the training set. Top: "Play." Bottom row: "No play."

motion measurements are presented in Fig. 7. Two-dimensional histograms of the camera-motion flow vectors are given in
Fig. 8. These histograms appear to be different enough, which confirms that the considered measurements of residual and camera motions are appropriate to distinguish the selected classes. Fig. 9 allows us to compare the ground truth and the results obtained with our recognition method. A few detection errors occur concerning video segments between closeup and long shot. However, the obtained results are globally accurate with a precision rate of between 0.86 and 0.90 and a recall rate of about 0.90 .

\section{Tennis Videos}

We have processed two tennis video data sets corresponding to two different tennis tournaments. 


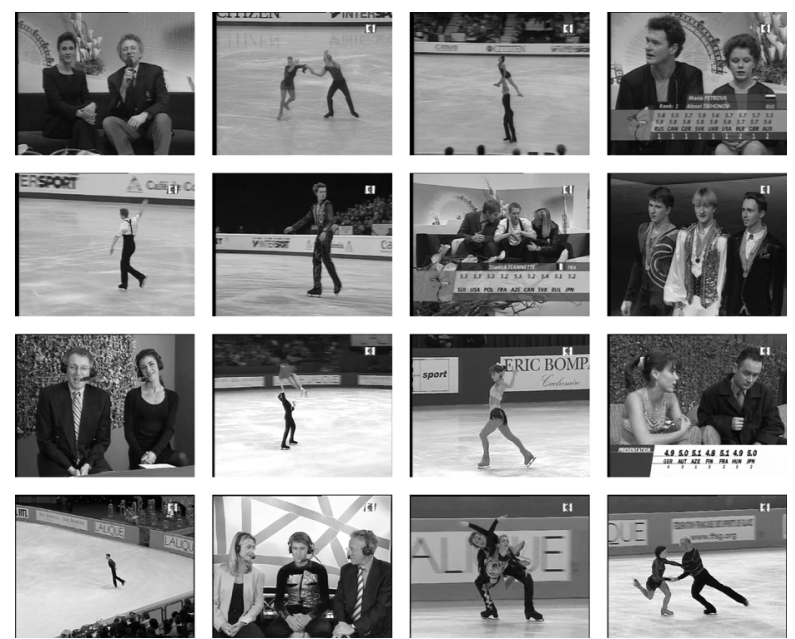

Fig. 6. Skating videos. Images extracted from the four videos used as test sets.

TABLE I

SKating Videos. Numbers of IMAGES FOR EACH PROCESSED VideO and Results of the Play/No Play Selection SteP (PRECISION AND RECALL FOR THE "PlAY" GROUP)

\begin{tabular}{|c|c|c|c|c|}
\hline & Video 1 & Video 2 & Video 3 & Video 4 \\
\hline \hline Number of images & 105600 & 83500 & 77000 & 100000 \\
\hline \hline Precision & 0.95 & 0.91 & 0.93 & 0.92 \\
\hline Recall & 0.65 & 0.72 & 0.68 & 0.62 \\
\hline
\end{tabular}
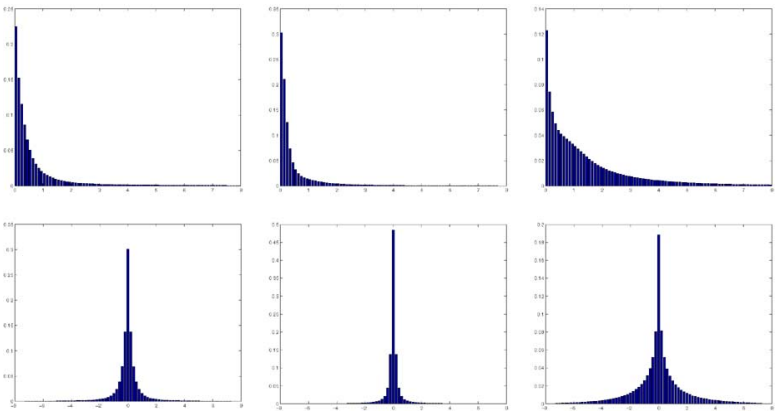

Fig. 7. Athletics video. Left to right: Pole vault shot, long shot of track race, and closeup of track race. Top row: Histograms of residual motion measurements. Bottom: Histograms of temporal contrasts of the residual motion measurements. (Color version available online at http://ieeexplore.ieee.org.)

1) French Open Tournament: We have applied our dynamic-content recognition method to a tennis TV program corresponding to the French open tournament. The first $42 \mathrm{~min}$ (63000 images) of the video are used as the training set (22 min for the learning of the motion models involved in the two stages and $20 \mathrm{~min}$ for the learning of outlier motion models), and the last 15 min (18000 images) form the test set. For this first experiment on tennis TV program, a manual temporal video segmentation is used in order to assess the performance of the recognition method by itself.

- Selecting play segments:

We want to select the "play" segments corresponding to the two tennis players in action and to discard the "no play" segments including views of the audience, referee shots or shots of the players resting, as illustrated in Fig. 10. We only exploit the first subset of the training set to learn
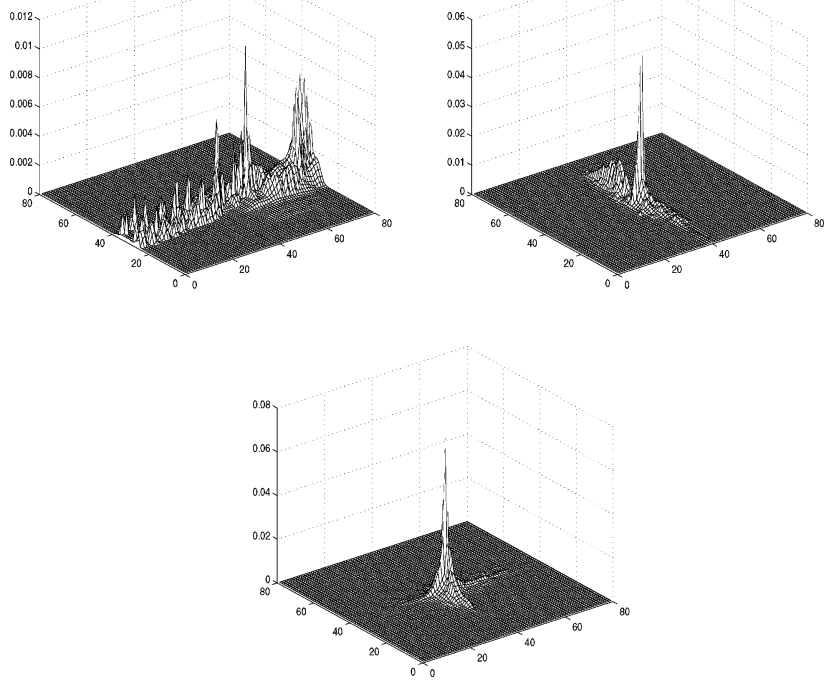

Fig. 8. Athletics video. Two-dimensional histograms of the camera-motion flow vectors. Left to right and top to bottom: Pole vault shot, long shot of track race, and closeup of track race.

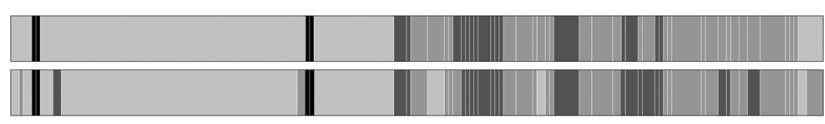

Fig. 9. Athletics video. Top row: Ground truth. Bottom row: Results of the dynamic-content recognition algorithm. From dark to bright: Pole vault, long shot of track race, closeup of track race, and no-play segments.
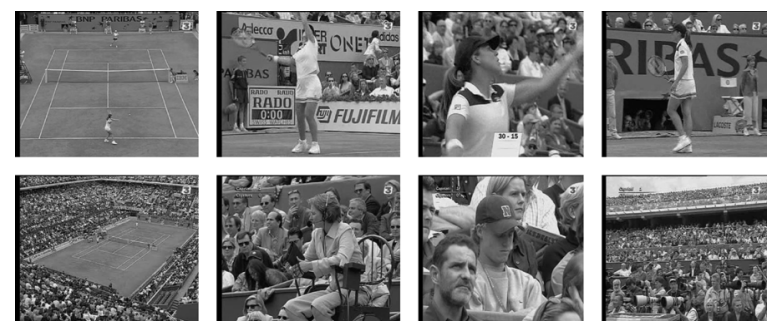

Fig. 10. French Open video. Top row: Image samples extracted from the group of "play" segments. Bottom row: Image samples extracted from "no play" segments.

the residual motion models that we need for this selection stage. 205 video segments of the training set represent "play" segments and 95 are "no play" segments. 31 residual motion clusters and their associated models are supplied by the AHC algorithm for the "play" group, and nine for the "no play" group. The large number of clusters obtained reveals the high diversity of dynamic contents in the two groups of the processed video.

Quite satisfactory results are obtained, since the precision rate for the play group is 0.88 and the recall rate is 0.89 .

- Detecting relevant events:

The goal is now to detect the relevant events of the tennis video among the segments selected as "play" segments. For this second stage, we introduce the probabilistic camera motion models. The three events we aim at detecting are the following: rally, serve, and change of side. In practice, we consider two subclasses for the serve class, which are wide-shot of serve and closeup of serve. Two 
TABLE II

FRENCH OPEN VIDEO. RESUlTS OF THE EVENT DETECTION TASK (PRECISION AND RECALL)

\begin{tabular}{|l|c|c|c|}
\hline & Rally & Serve & Change of side \\
\hline Precision & 0.92 & 0.63 & 0.85 \\
\hline Recall & 0.89 & 0.77 & 0.74 \\
\hline
\end{tabular}
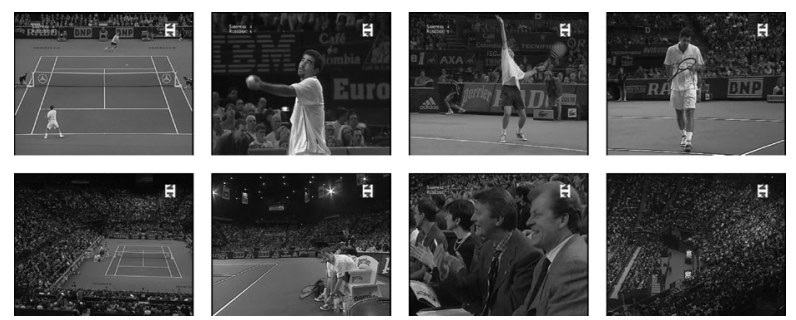

Fig. 11. Paris-Bercy'98 tournament. Top row: Image samples extracted from the group of "play" segments. Bottom row: Image samples extracted from "no play" segments.
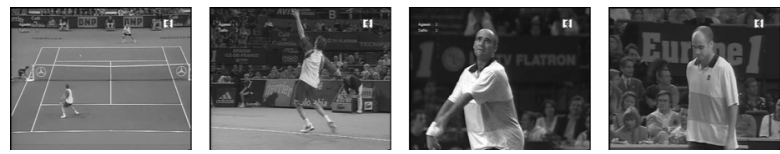

Fig. 12. Images extracted from the final of the Paris-Bercy'99 tournament.

subclasses are considered also for the change-of-side class. As a consequence, five residual motion models and five camera motion models have to be learned. We have also to determine the residual motion models accounting for the outlier segments for each class. Results are reported in Table II. Satisfactory results are obtained, especially for the rally class. The precision rate of the serve class is lower than the others. In fact, for the serve class, errors come from the play/no play selection stage (i.e., some serve segments are wrongly put in the "no play" group, and then, are lost). Let us point out that the recall rate is computed over all the video segments of the given class in the original video and not in the play group only. It appears that a few serve segments are difficult to distinguish from some "no play" segments when using only motion information. However, the proposed statistical framework could easily integrate other information such as color or audio (ball hits, applause) as in [39], to improve this classification rate.

2) Paris-Bercy Tournament: We have also processed two other tennis TV programs corresponding to two finals of ParisBercy indoor tournament in 1998 and in 1999. The first tennis video "Paris-Bercy'98" contains 188895 images (i.e., the video length is 2h06). The first 99085 images are used as the training set (63 490 images for the learning of the motion models involved in the selection and event recognition stages and 35595 images for the learning of outlier models). The last $89910 \mathrm{im}$ ages (i.e., one hour) form our first test set. Our second test set is supplied by the second video "Paris-Bercy'99" which includes another tennis game between two different players. No updating of the learning step has been performed. This video consists of 246500 images, that is, the video length is $2 \mathrm{~h} 44$. Some images extracted from the two videos are displayed in Figs. 11 and 12.
TABLE III

PARIS-Bercy TOURNAMENT. NuMBER OF VIDEO SEgMENTS For THE Two Test Sets in Each Event Class. Test Set 1: End of the Final of PARIS-Bercy'98. TeSt Set 2: Final OF Paris-Bercy'99

\begin{tabular}{|c|c|c|c|}
\hline & Rally & Serve & Change of side \\
\hline Test set 1 & 66 & 38 & 139 \\
\hline Test set 2 & 327 & 218 & 461 \\
\hline
\end{tabular}

TABLE IV

Paris-Bercy Tournament. Results of the Play/No Play Selection STEP (PReCision and ReCAll FOR the "Play" Group). Test SET 1: END OF THE FINAL OF PARIS-BERCY'98. TEST SET 2: FINAL OF PARIS-BERCY'99

\begin{tabular}{|c|c|c|}
\hline & Test set 1 & Test set 2 \\
\hline Precision & 0.90 & 0.84 \\
\hline Recall & 0.88 & 0.90 \\
\hline
\end{tabular}

TABLE V

PARIS-BERCy TOURnAMENT. RESUlTS OF THE EvENT DETECTION TASK. Top: TeSt Set 1. BotTom: TeSt SeT 2

\begin{tabular}{|c|c|c|c|}
\hline & Rally & Serve & Change of side \\
\hline Precision & 0.94 & 0.34 & 0.88 \\
\hline Recall & 0.94 & 0.58 & 0.73 \\
\hline \multicolumn{4}{|c|}{ Test set 1} \\
\hline
\end{tabular}

\begin{tabular}{|c|c|c|c|}
\hline & Rally & Serve & Change of side \\
\hline Precision & 0.85 & 0.38 & 0.82 \\
\hline Recall & 0.95 & 0.46 & 0.79 \\
\hline \multicolumn{4}{|c|}{ Test set 2 }
\end{tabular}

These two videos have been automatically segmented into homogeneous temporal units using the motion-based method described in [19]. We have added a postprocessing step to eliminate the smallest segments (less than 25 frames).

- Selecting video segments:

The "play" group and the "no play" groups are defined as mentioned above in Section VIII-C1. We only exploit the first subset of the training set to learn the residual motion models that we need for the selection step; 109 video segments of the training set are "play" segments and 31 are "no play" segments. Table IV shows the good performance of the selection step for these two test sets while an automatic video segmentation is employed.

- Detecting relevant events:

We aim at detecting the same three events: Rally, Serve and Change of side among the segments previously selected as "play" segments. Let us recall that the probabilistic models used are learned from the beginning of the "Paris-Bercy'98" game. The obtained results for the two test sets (end of the "Paris-Bercy'98" game and "ParisBercy'99" game) are given in Table V. Similar precision and recall rates are obtained for the two test sets which demonstrates the robustness of our method. The rally and the change of side events are correctly detected, but the performance is drastically decreases for the serve class. This can be partly explained by the different temporal video segmentation used for the two experiments (manual for the French open video and automatic for Paris-Bercy videos). For Paris-Bercy videos, the automatic temporal segmentation has a tendency to oversegmentation. A lot of obtained serve segments are too small (less than 25 frames) and are eliminated. Therefore, as reported in Table III, there are few serve segments. Moreover, small rally and change of 
side segments are wrongly labeled as serve. This explains the obtained rates for the serve class. Again adding audio information would greatly improve the classification rate [39].

\section{CONCLUSION}

We have proposed a novel statistical framework for recognizing motion content in videos without any available predefined motion models and for non restricted situations. We have demonstrated its interest for event detection in sports videos. We have introduced new probabilistic motion models representing the scene motion and the camera motion, respectively. These models are quite parsimonious (they involve very few parameters) while being able to adequatly discriminate different dynamic video contents. They can be easily learned from the image sequence and can handle a large variety of dynamic video contents. The proposed residual motion model is a global occurrence model. Consequently, it does not inform on the spatial and temporal arrangements of these local motion measurements. A finer event or action categorization could require such a complementary information. Developments are actually in progress to extend these motion models in that direction, i.e., adding spatial context [40], and accounting for temporal causality [41]. The design of local probabilistic motion models (i.e., the same type of models but applied to selected space-time blocks) is also investigated.

We have reported a large set of experiments on sports TV programs demonstrating the accuracy and the robustness of the proposed approach and its capacity to achieve supervised event recognition tasks. Moreover, it is flexible enough to properly introduce prior on the event classes if available, or to incorporate other kinds of video primitives (such as color or audio ones) that are obviously of interest if dealing with a full video indexing or summarization framework (which was not the scope of this paper). The proposed two-step method for dynamic-content recognition is general and does not exploit very specific knowledge (related to the video genre, e.g., type of sport) and dedicated solutions. It could be applied to a large range of videos.

\section{ACKNOWLEDGMENT}

The authors would like to thank INA for providing the video sequences.

\section{REFERENCES}

[1] N. Haering and N. da Vitoria Lobo, Visual Event Detection, ser. The International Series in Video Computing. New York: Springer, 2001, vol. 2.

[2] O. Faugeras, Three-Dimensional Computer Vision, a Geometric Viewpoint. Cambridge, MA: MIT Press, 1993.

[3] D. Gavrila, "The visual analysis of human movement: a survey," Comput. Vis. Image Understand., vol. 73, no. 1, pp. 82-98, 1999.

[4] G. Doretto, A. Chiuso, Y. Wu, and S. Soatto, "Dynamic textures," Int. J. Comput. Vis., vol. 51, no. 2, pp. 91-109, 2003.

[5] P. Bouthemy, M. Gelgon, and F. Ganansia, "A unified approach to shot change detection and camera motion characterization," IEEE Trans. Circuits Syst. Video Technol., vol. 9, no. 7, pp. 1030-1044, Jul. 1999.
[6] H. Greenspan, J. Goldberger, and A. Mayer, "Probabilistic space-time video modeling via piecewise GMM," IEEE Trans. Pattern Anal. Mach. Intell., vol. 26, no. 3, pp. 384-396, Mar. 2004.

[7] E. Bruno and D. Pellerin, "Video structuring, indexing and retrieval based on global motion wavelet coefficients," in Proc. 16th Int. Conf. Pattern Recognition, Quebec City, QC, Canada, Aug. 2002, vol. 3, pp. 287-290.

[8] C.-W. Ngo, T.-C. Pong, and H.-J. Zhang, "On clustering and retrieval of video shots through temporal slices analysis," IEEE Trans. Multimedia, vol. 4, no. 4, pp. 446-458, Aug. 2002.

[9] L. Zelnik-Manor and M. Irani, "Event-based video analysis," in Proc. IEEE Conf. Computer Vision and Pattern Recognition, Kauai, HI, Dec. 2001, vol. 2, pp. 123-130.

[10] I. Laptev and T. Lindeberg, "Local descriptors for spatio-temporal recognition," presented at the Int. Workshop Spatial Coherence for Visual Motion Analysis, Prague, Czech Republic, May 2004.

[11] Y. Rui and P. Anandan, "Segmenting visual actions based on spatiotemporal motion patterns," in Proc. IEEE Conf. Computer Vision and Pattern Recognition, Hilton Head, SC, Jun. 2000, vol. 1, pp. 111-118.

[12] E. Ardizzone, M. L. Cascia, A. Avanzato, and A. Bruna, "Video indexing using MPEG motion compensation vectors," in Proc. IEEE Int. Conf. Multimedia Computing and Systems, Florence, Italy, Jun. 1999, vol. 2, pp. 725-729.

[13] Y. Deng and B. Manjunath, "Content-based search of video using color, texture and motion," in Proc. 4th IEEE Int. Conf. Image Processing, Santa Barbara, CA, Oct. 1997, pp. 543-547.

[14] F. Bashir, A. Khokhar, and D. Schonfeld, "Object trajectory-based activity classification and recognition using hidden Markov models," IEEE Trans. Image Process., 2005, to be published.

[15] A. Ekin, A. Tekalp, and R. Mehrotra, "Automatic soccer video analysis and summarization," IEEE Trans. Image Process., vol. 12, no. 7, pp. 796-807, Jul. 2003.

[16] H. Denman, N. Rea, and A. Kokaram, "Content-based analysis for video from snooker broadcasts," Comput. Vis. Image Understand., vol. 92, pp. 176-195, 2003.

[17] A. Hanjalic, "Generic approach to highlights extraction from a sport video," presented at the 10th IEEE Int. Conf. Image Processing, Barcelona, Spain, Sep. 2003.

[18] R. Leonardi, P. Migliorati, and M. Prandini, "Semantic indexing of soccer audio-visual sequences: A multi-modal approach based on controlled Markov chains," IEEE Trans. Circuits Syst. Video Technol., vol. 14, no. 5, pp. 634-643, May 2004.

[19] F. Coldefy and P. Bouthemy, "Unsupervised soccer video abstraction based on pitch, dominant color and camera motion analysis," presented at the 12th ACM Int. Conf. Multimedia, New York, Oct. 2004.

[20] N. Babaguchi, Y. Kawai, T. Ogura, and T. Kitahashi, "Personalized abstraction of broadcasted american football video by highlight selection," IEEE Trans. Multimedia, vol. 6, no. 4, pp. 575-585, Aug. 2004.

[21] J. Fan, H. Luo, and A. Elmagarmid, "Concept-oriented indexing of video databases: toward semantic sensitive retrieval and browsing," IEEE Trans. Image Process., vol. 13, no. 7, pp. 974-992, Jul. 2004.

[22] J. Assfalg, M. Bertini, A. D. Bimbo, W. Nunziati, and P. Pala, "Soccer highlights detection and recognition using HMMs," in IEEE Int. Conf. Multimedia and Expo, Lausanne, Switzerland, Aug. 2002.

[23] P. Chang, M. Han, and Y. Gong, "Extract highlights from baseball game video with hidden Markov models," presented at the 9th IEEE Int. Conf. Image Processing, Rochester, NY, Sep. 2002.

[24] X. Gibert, H. Li, and D. Doermann, "Sports video classification using HMMs," presented at the IEEE Int. Conf. Multimedia and Expo, Baltimore, MD, 2003.

[25] R. Leonardi and P. Migliorati, "Semantic indexing of multimedia documents," IEEE Trans. Multimedia, vol. 9, no. 2, pp. 44-51, Apr. 2002.

[26] Y. Wang, Z. Liu, and J. Huang, "Multimedia content analysis using both audio and visual clues," IEEE Signal Process. Mag., vol. 17, no. 6, pp. 12-36, Jun. 2000.

[27] N. Vasconcelos and A. Lippman, "Statistical models of video structure for content analysis and characterization," IEEE Trans. Image Process., vol. 9, no. 1, pp. 3-19, Jan. 2000.

[28] _ - "A bayesian framework for semantic content characterization," in Proc. IEEE Conf. Computer Vision and Pattern Recognition, Santa Barbara, CA, Jun. 1998, pp. 566-571.

[29] J. Hoey and J. Little, "Bayesian clustering of optical flow fields," in Proc. 9th IEEE Int. Conf. Computer Vision, Nice, France, Oct. 2003, pp. 1086-1093. 
[30] F. Wang, Y.-F. Ma, H.-J. Zhang, and J.-T. Li, "A generic framework for semantic sports video analysis using dynamic bayesian networks," presented at the 11th Int. Multimedia Modelling Conf., Melbourne, Australia, Jan. 2005.

[31] M. Barnard and J.-M. Odobez, "Sports event recognition using layered HMMs," presented at the Int. Conf. Multimedia and Expo, Amsterdam, The Netherlands, 2005.

[32] G. Piriou, P. Bouthemy, and J.-F. Yao, "Extraction of semantic dynamic content from videos with probabilistic motion models," presented at the 8th Eur. Conf. Computer Vision, Prague, Czech Republic, May 2004.

[33] R. Fablet and P. Bouthemy, "Motion recognition using non parametric image motion models estimated from temporal and multiscale cooccurrence statistics," IEEE Trans. Pattern Anal. Mach. Intell., vol. 25, no. 12, pp. 1619-1624, Dec. 2003.

[34] J.-M. Odobez and P. Bouthemy, "Robust multiresolution estimation of parametric motion models," J. Vis. Commun. Image Represent., vol. 6, no. 4 , pp. $348-365,1995$.

[35] M. Irani, B. Rousso, and S. Peleg, "Detecting and tracking multiple moving objects using temporal integration," in Proc. 2nd Eur. Conf. Computer Vision, May 1992, vol. 588, pp. 282-287.

[36] J.-M. Odobez and P. Bouthemy, "Detection of multiple moving objects using multiscale MRF with camera motion compensation," presented at the 1st IEEE Conf. Image Processing, Austin, TX, Nov. 1994.

[37] N. Peyrard and P. Bouthemy, "Detection of meaningful events in videos based on a supervised classification approach," presented at the 10th IEEE Int. Conf. Image Processing, Barcelona, Spain, Sep. 2003.

[38] C. Biernacki, G. Celeux, and G. Govaert, "Assessing a mixture model for clustering with the integrated completed likelihood," IEEE Trans. Pattern Anal. Mach. Intell., vol. 22, no. 3, pp. 719-725, Mar. 2000.

[39] F. Coldefy, P. Bouthemy, M. Betser, and G. Gravier, "Tennis video abstraction from audio and visual cues," presented at the IEEE Int. Workshop Multimedia Signal Processing, Sep. 2004.

[40] P. Bouthemy, C. Hardouin, G. Piriou, and J.-F. Yao, "Mixed-state automodels and motion texture modeling," J. Math. Imag. Vis., to be published.

[41] G. Piriou, P. Bouthemy, and J.-F. Yao, "Motion content recognition in video database with mixed-state probabilistic causal models," presented at the Workshop on Content-Based Multimedia Indexing, Aug. 2006, online.

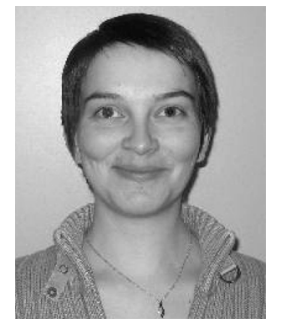

Gwenaélle Piriou was born in 1976. She received the Ph.D. degree in signal processing and telecommunications from the University of Rennes, France, in 2005.

She is currently a Lecturer with the computer science department of University of Rennes. Her main research interests are statistical modeling for image sequence analysis, learning, and recognition and classification of dynamic video contents in video.

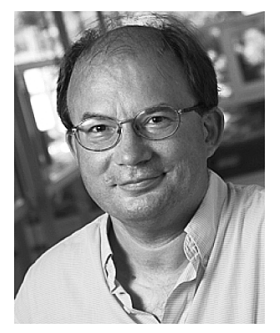

Patrick Bouthemy graduated from ENST, Paris, in 1980, and received the Ph.D. degree in computer science from the University of Rennes, Rennes, France, in 1982.

From 1983 to 1984 , he was with the Department of Visual Communications, INRS-Telecommunications, Montreal, QC, Canada. Since 1984, he has been with INRIA, IRISA, Rennes. He is currently "Directeur de Recherche" at INRIA and Head of the Vista group. His main research interests are: statistical approaches for image sequence processing, image sequence denoising, motion detection, segmentation and estimation, learning, and recognition and classification of dynamic contents in video. $\mathrm{He}$ has served as member of the program committees of the major conferences in image processing and computer vision.

Dr. Bouthemy was an Associate Editor of the IEEE TRANSACTIONS ON IMAGE PROCESSING from 1999 to 2003.

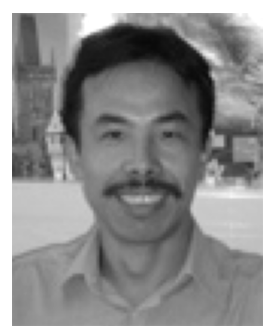

Jian-Feng Yao received the Ph.D. degree in applied mathematics from the Université Paris-Sud, Orsay, France, in 1990.

From January 1990 to August 2000, he was a Maître de Conference at the University of Paris I, Paris, France. Since September 2000, he has been a Full Professor of statistics at the University of Rennes I, Rennes, France. His current research interests are nonlinear time series analysis, spectral theory of large-dimensional random matrices, and mathematical modeling for image understanding. 